\title{
Phase stability of fluorinated nanodiamonds under HPHT treatment
}

\author{
V.N. Khabashesku ${ }^{1}$, V.P. Filonenko ${ }^{2}$, A. S. Anokhin $^{3}$, E. V. Kukueva ${ }^{4}$ \\ ${ }^{1}$ Center for Technology Innovation, Baker Hughes a GE Company, Houston, TX, USA \\ ${ }^{2}$ Vereshchagin Institute for High Pressure Physics RAS, Moscow, Troitsk, Russia \\ ${ }^{3}$ A. A. Baikov Institute of Metallurgy and Materials Science RAS, Moscow, Russia \\ ${ }^{4}$ Lomonosov Moscow State University, Moscow, Russia \\ valery.khabashesku@bakerhughes.com, filv@hppi.troitsk.ru, alexanderanokhin@yahoo.com, \\ elena.kukueva@gmail.com
}

PACS 62.50.-p, 81.07.-b, 81.05.ug

DOI 10.17586/2220-8054-2018-9-1-25-28

\begin{abstract}
The aspects of phase and size stability of surface fluorinated nanoscale diamond powders during their treatment under conditions of high pressures and high temperatures (HPHT) are considered. In the studied powder, fluorine is covalently bonded to diamond particles, replacing the other functional groups on their surface. In this case, under pressure of $8.0 \mathrm{GPa}$ the transition of 10-nm-size diamond nanoparticles into a graphene layered carbon forms does not occur up to temperatures of $1700^{\circ} \mathrm{C}$, and their size does not change. The addition of submicro-sized aluminum powder to fluorinated nanodiamond results in the fast growth of particles to a micrometer size range. The observed unprecedented enlargement of nanodiamonds to micro-sized crystals is explained by occurrences of Wurtz-type reactions in the C-Al-F system which activate the formation of new interfacial carbon-carbon bonds between nanoparticles and their coalescence under HPHT conditions.
\end{abstract}

Keywords: fluorinated nanodiamonds, high pressure-high temperature treatment, aluminum fluoride.

Received: 13 June 2017

Revised: 7 December 2017

\section{Introduction}

Nanocarbons tend to undergo different phase transformations depending on particle size. Studies of the relationships between ensembles of nanoparticles under $\mathrm{P}-\mathrm{T}$ conditions of stability of diamond phase on carbon diagram are of particular interest. The phase transformation pathway depends on thermodynamic conditions, size and morphology of the nanoparticles and composition of surface contaminants. As an example, globular turbostratic carbon was shown to transform into diamond nanocrystals through a reordering of multi-layered carbon structures under HPHT treatment at pressure of $8.0 \mathrm{GPa}$ in the temperature range of $1300-1500{ }^{\circ} \mathrm{C}$ [1]. On contrary, under the same $\mathrm{P}-\mathrm{T}$ parameters, nanodiamonds transform into graphite after reaching an average particle size of $20 \mathrm{~nm}[2]$.

Important role in the kinetics of crystallization and growth of diamonds under high and low pressures is being played by hydrogen. It is known that the HPHT treatment of hydrocarbons at $8 \mathrm{GPa}$ and $1300{ }^{\circ} \mathrm{C}$ leads to the formation of micro-sized diamond crystals [3], while the addition of naphthalene $\left(\mathrm{C}_{10} \mathrm{H}_{8}\right)$ to detonation nanodiamonds activates particle growth under similar thermobaric conditions [4]. In the $\mathrm{C}-\mathrm{H}-\mathrm{F}$ system, both micro- and nanodiamonds are formed under HPHT treatment. On the example of thermobaric action on $\mathrm{C}_{10} \mathrm{H}_{8^{-}}$ $\mathrm{C}_{10} \mathrm{~F}_{8}$ mixture, the mechanism of such tranformations has been interpreted by simultaneous generation of two types of carbon precursors - non-graphitic nano-sized and graphitic micro-sized particles [5-7]. The micro-sized diamond fractions are formed directly from graphitic precursors, while the nano-sized fraction of diamond is formed from $5-15 \mathrm{~nm}$ onion-like carbon nanoparticles. An important role in the transformation processes is played by the gas-phase or fluid-phase transport of carbon and surface chemical reactions. In comparison, the formation of diamonds from fluorographite $\left(\mathrm{CF}_{1.1}\right)$ at $8.0 \mathrm{GPa}$ was not observed at all within the whole range of the temperatures studied, up to $1500{ }^{\circ} \mathrm{C}$. The present work shows the effect of HPHT treatment on phase stability of fluorinated nanodiamonds (FND).

\section{Experimental section}

The diamond nanopowder, comprised of $10 \mathrm{~nm}$ average size particles of spherical shape, has been produced by detonation method followed by high energy grinding to crush the tightly bonded agglomerates. Fluorinated nanodiamonds (FND10), containing $7 \mathrm{wt} \%$ of fluorine covalently bonded to carbon atoms on the surface, were obtained by $\mathrm{F}_{2}-\mathrm{He}$ treatment of the nanopowder at $340{ }^{\circ} \mathrm{C}$. Aluminum powder, comprised of spherical particles of 
$0.8 \mu \mathrm{m}$ average size, was used as an additive to diamond powder. Powders were mixed in the ethanol media with the aid of ultrasound.

HPHT treatment of samples was carried out under pressure of $8.0 \mathrm{GPa}$ and different temperatures up to $1700{ }^{\circ} \mathrm{C}$ in the Toroid-type cell. The pressure was generated with the tungsten carbide anvils by a uniaxial hydraulic system. Samples (5 mm diameter, $3 \mathrm{~mm}$ height) were quenched under pressure, recovered at ambient conditions, and then characterized by X-ray diffraction, Raman spectroscopy, scanning (SEM) and transmission (TEM) electron microscopies.

\section{Results and discussion}

It was found that fluorinated diamond nanoparticles do not transform into graphite structures after treatment at the pressure of $8.0 \mathrm{GPa}$, a temperature of $1500{ }^{\circ} \mathrm{C}$ and an isothermal exposure time of $60 \mathrm{~s}$ (Fig. 1). The TEM images on Fig. 1 show that after thermobaric treatment, the size of FND crystals did not change while the electron diffraction pattern after the HPHT treatment looks more diffused in comparison with the initial material. The Raman spectra (not shown) of the high pressure compacts differentiated from the starting fluorinated powder by only a slight broadening and shifting of the peaks' maxima. Reflections due to the graphite phase were also not observed in the XRD of compacts. Elemental analysis of the fracture surface of the compact has indicated that fluorine content is several times reduced, to $1.5-2.0 \mathrm{wt} \%$, after the thermobaric treatment of FND10.
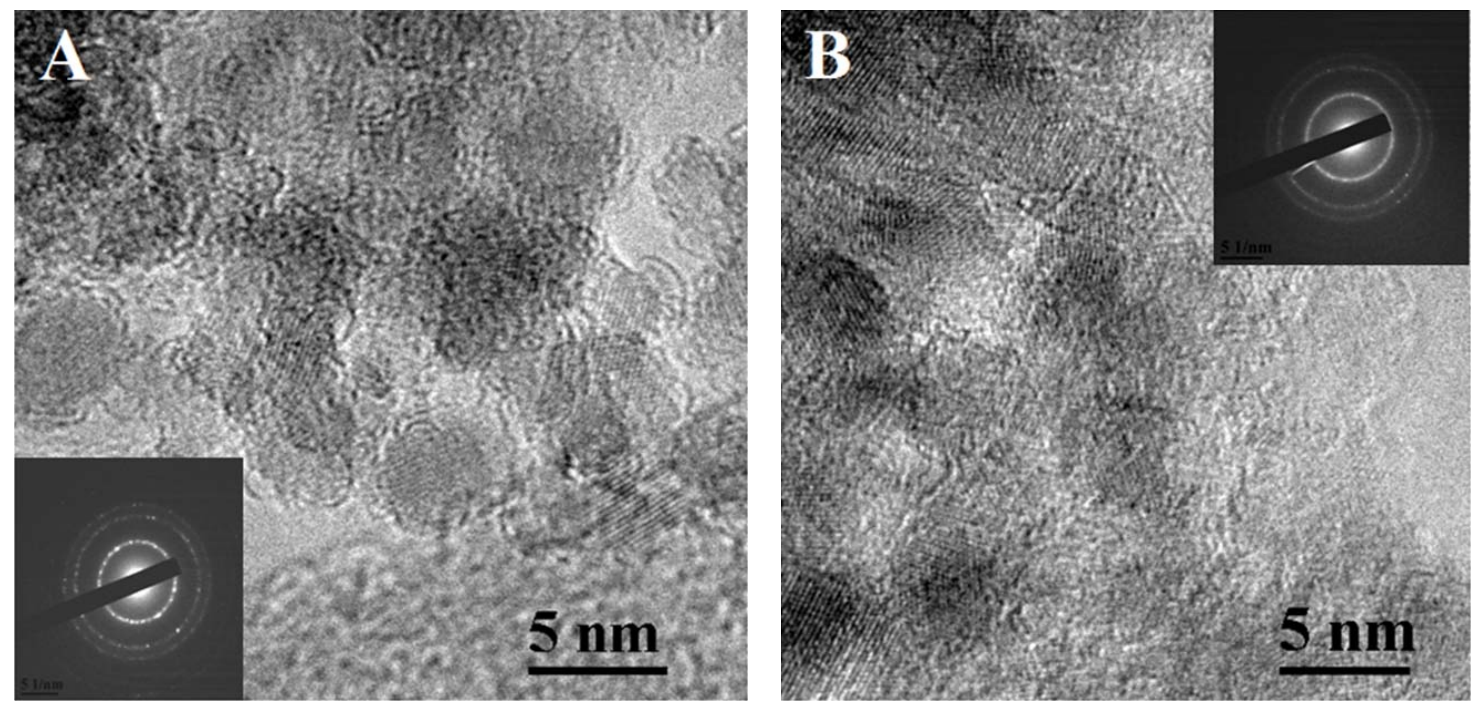

FIG. 1. TEM images of fluorinated diamond nanopowder taken before (A) and after HPHT treatment (B)

During heating at temperatures above $700{ }^{\circ} \mathrm{C}$ fluorine detaches from diamond surface and then exists within the volume of compact in the form of inert fluid which does not provide a mass transfer of carbon which is needed for growth of nanodiamond particles. It should be noted that the thermobaric treatment of a non-fluorinated nanodiamond powder under the same pressure and temperature conditions has led to its substantial graphitization. Thus, the presence of fluorine on the surface of diamond nanoparticles helps to stabilize the cubic structure and slow down the process of graphitization.

Aluminum submicropowder (up to $15 \mathrm{wt} \%$ ) was admixed to FND10 nanopowder with the idea of scavenging and chemically trapping and binding the fluorine in the volume of HPHT treated fluorinated nanodiamond compact sample. In this case, the void-free grain boundaries were observed to form between nanodiamond particles along with the formation of aluminum fluoride. As a result, an unprecedented growth and enlargement of diamond crystals from nano to micro sizes was discovered to occur in this system on HPHT treatment at $1700{ }^{\circ} \mathrm{C}$. In addition to that, a complete absence of formation of even trace amounts of graphite phase has been noted.

From the diffraction patterns in Fig. 2, it is seen that at temperatures around $1600{ }^{\circ} \mathrm{C}$ a sharp peak, characteristic of micrometer-sized diamond particles, appears on the top of the initially broad peak of nanodiamond. If the temperature of the thermobaric treatment is increased to $1700^{\circ} \mathrm{C}$, all nanodiamonds become fused into crystals of micrometer size. They do not show any external signs of conservation of the nanostructured state. The morphology of diamond particles obtained after the thermobaric treatment at $1700{ }^{\circ} \mathrm{C}$ is shown in Fig. 3 . 


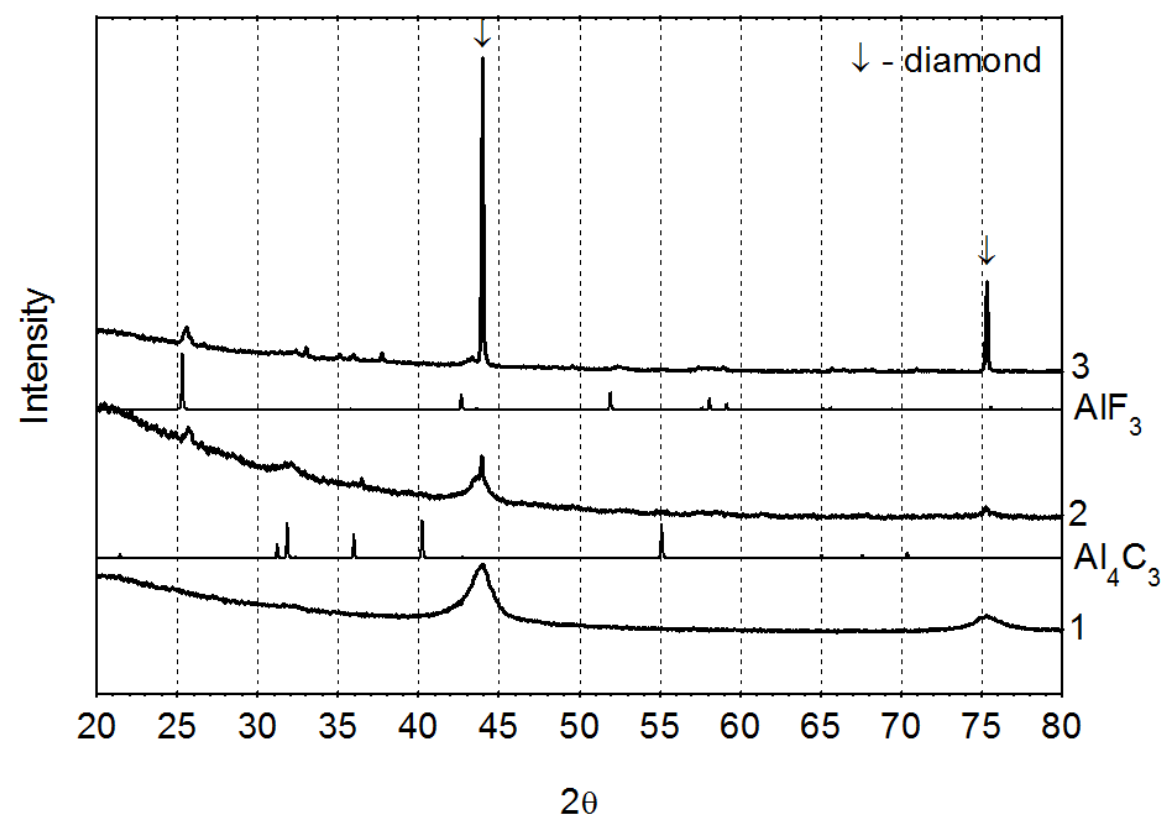

FIG. 2. X-ray analysis of mixtures FND10-15Al: 1 - nanopowder FND10 before termobaric treatment; 2 - mixture FND10-15Al after treatment at $7.5-8.0 \mathrm{GPa}, 1550-1600{ }^{\circ} \mathrm{C}, 30 \mathrm{~s} ; 3-$ mixture FND10-15Al after treatment at $7.5-8.0 \mathrm{GPa}, 1650-1700{ }^{\circ} \mathrm{C}, 30 \mathrm{~s}$

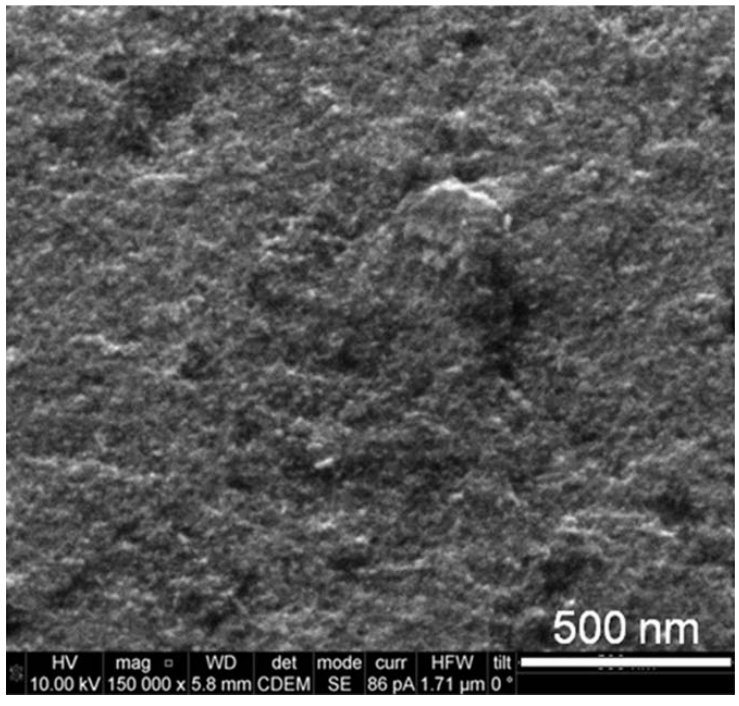

(A)

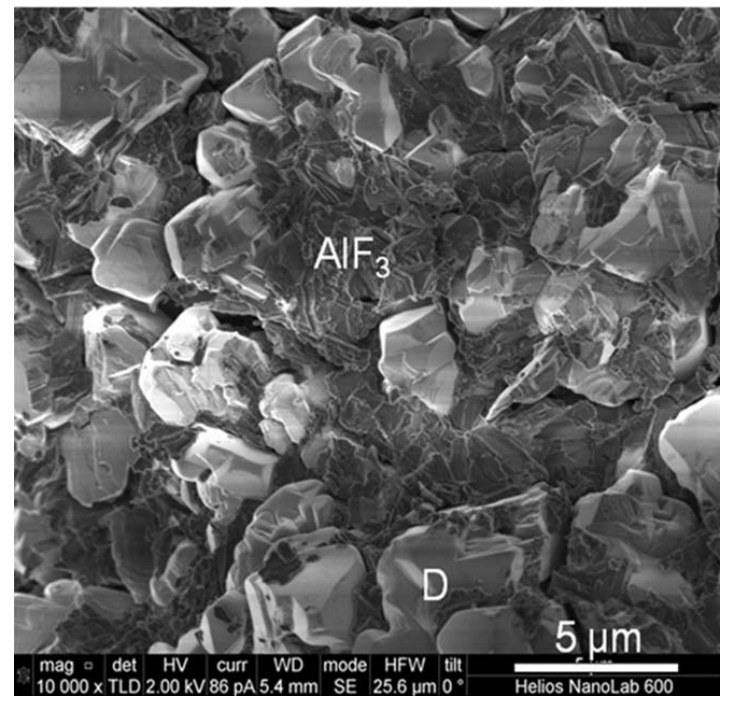

(B)

FIG. 3. SEM images of initial (A) and treated at $7.5-8.0 \mathrm{GPa}, 1650-1700^{\circ} \mathrm{C}, 30 \mathrm{~s}(\mathrm{~B})$ mixtures FND10-15Al

The micro-sized diamond crystals, formed from the nanoparticles, exhibit well-faceted shapes and are separated by aluminum fluoride. It should be noted that the process of unusual growth and enlargement of nanodiamonds to the micro-sized species observed in the $\mathrm{C}-\mathrm{Al}-\mathrm{F}$ system occurs in a quite narrow temperature range. This is partly determined by the thermodynamics, namely the tendency of the system to reduce energy by reducing the total surface of the interfacial grain boundaries. Temperatures of $1600-1700{ }^{\circ} \mathrm{C}$ and pressures not lower than $7.5 \mathrm{GPa}$ are required for the realization of the growth process in the whole volume of the sample. An increase in temperature or decrease in pressure leads to a partial transition of nano- and microdiamonds to a graphite phase. It is seen from the diffraction pattern that aluminum fluoride is required for the enlargement growth of nanodiamonds. Aluminum fluoride melts at a temperature of about $1300{ }^{\circ} \mathrm{C}$. Under the applied thermobaric treatment conditions, it is in a liquid state, and in such state, it can activate the transport of carbon from the surface of diamond nanoparticles. 
Therefore, the anomalous growth of nanodiamonds up to the micro-sized diamond crystals observed in the present work, can be associated with both the realization of Wurtz-type reaction under the HPHT conditions and with the formation of the fluid Al-C-F phases in the samples volume, accelerating the mass transport and re-crystallization of nanodiamonds.

\section{References}

[1] Filonenko V., Zibrov I., et al. Structural and morphological transformations of nanosized globular carbon during thermobaric treatment. Inorg. Mater, 2017, 53 (5), P. 1-8.

[2] Davydov V., Rakhmanina A., Agafonov V., Khabashesku V. Size-dependent nanodiamond-graphite phase transition at 8 GPa. JETP Lett., 2007, 86 (7), P. 462-464.

[3] Davydov V.A., Rakhmanina A.V., et al. Conversion of polycyclic aromatic hydrocarbons to graphite and diamond at high pressures. Carbon, 2004, 42, P. 261-269.

[4] Varfolomeeva T.D., Lyapin A.G., et al. Behavior of detonation nanodiamond at high pressures and temperatures in the presence of a hydrogen-containing fluid. Inorg. Mater, 2016, 52 (4), P. 351-356.

[5] Davydov V.A., Rakhmanina A.V., Agafonov V.N., Khabashesku V.N. Synergistic effect of fluorine and hydrogen on processes of graphite and diamond formation from fluorographite-naphthalene mixtures at high pressures. J. Phys. Chem. C, 2011, 115, P. 21000-21008.

[6] Davydov V.A., Rakhmanina A.V., Agafonov V.N., Khabashesku V.N. On the nature of simultaneous formation of nano- and micro-size diamond fractions under pressure-temperature-induced transformations of binary mixtures of hydrocarbon and fluorocarbon compounds. Carbon, 2015, 90, P. 231-233.

[7] Davydov V.N., Agafonov V.N., Khabashesku V.N. Comparative study of condensation routes for formation of nano- and microsized carbon forms in hydrocarbon, fluorocarbon, and fluoro-hydrocarbon systems at high pressures and temperatures. J. Phys. Chem. C, 2016, 120 (51), P. 29498-29509. 